\begin{tabular}{|c|c|}
\hline Title & $\begin{array}{l}\text { A dvanced Bondarenko method for resonance self-shielding cal culations in deterministic reactor physics code system } \\
\text { CBZ }\end{array}$ \\
\hline Author(s) & Chiba, Go; Narabay ashi, Tadashi \\
\hline Citation & $\begin{array}{l}\text { A nnals of nuclear energy, } 96,277-286 \\
\text { https://doi.org/10.1016/.anucene.2016.06.009 }\end{array}$ \\
\hline Issue Date & $2016-10$ \\
\hline Doc URL & http:/hdl.handle.net/2115/71585 \\
\hline Rights & $\begin{array}{l}\text { (9 2016. This manuscript version is made available under the CC-BY-NC-ND } 4.0 \text { license } \\
\text { http://reativecommons.org/icenses/by-nc-nd/4.0/ }\end{array}$ \\
\hline Rights(URL) & http://creativecommons.org/icenses/by-nc-nd/4.0/ \\
\hline Type & article (author version) \\
\hline File Information & main_r1.pdf \\
\hline
\end{tabular}

Instructions for use 


\title{
Advanced Bondarenko method for resonance self-shielding calculations in deterministic reactor physics code system CBZ
}

\author{
Go Chiba*,a ${ }^{*}$ Tadashi Narabayashi ${ }^{\mathrm{a}}$ \\ ${ }^{a}$ Hokkaido University, Kita-ku, Sapporo, Hokkaido 060-8628, Japan
}

\section{${ }_{5}$ Abstract}

The advanced Bondarenko method for resonance self-shielding calculations is devised and proposed. This method is based on three numerical methods; the Bell factor optimization for accurate fuel escape probability representation, extension of resonance interference factors and correction factors for current-weighted total cross sections. A 107-group library for light water reactor applications based on the advanced Bondarenko method is generated for a reactor physics code system CBZ. Performance of the CBZ code with this 107-group library is examined against a suit of light water reactor cell problems. The infinite neutron multiplication factors calculated with CBZ agree with reference continuous-energy Monte Carlo solutions within $0.15 \% \Delta k / k k^{\prime}$ differences, and no significant biases on fuel compositions and geometrical specifications are observed. Energy-averaged cross sections are also examined. Numerical tests reveal that significant accuracy improvements in resonance self-shielding calculations are realized by adopting the advanced Bondarenko method without any significant increase of computational burden.

6 Key words: resonance self-shielding, Bondarenko method, resonance interference

\section{Introduction}

The deterministic method for nuclear fission reactors calculations has played important roles in the field 9 of nuclear engineering, and is expected to do that also in future. One of the most important subjects

*Corresponding author, Tel: +81-10-706-6683, Fax: +81-10-706-6683

Email address: go_chiba@eng.hokudai.ac.jp (Go Chiba) 
developed and proposed. The Bondarenko method(Bondarenko, 1964) is one of concepts of multi-group cross section libraries which are suited to calculations based on the equivalence principle.

In the past, reactor physics researchers and engineers had focused on how to improve the calculation accuracy of energy-averaged cross sections under a limited number of energy groups because of computational performance limitation. As the computer technology has been significantly advanced, this situation has been drastically changing in recent years, and the direct approaches, that are to increase the number of energy groups for explicit representations of wide resonances, have been rather preferred now. For example, the French APPOLLO code uses 281-group library(Sanchez, 2010) and the Studsvik CASMO code uses 586group library(Rhodes, 2012).

In the present paper, we attempt to improve the calculation accuracy of energy-averaged cross sections without any increase of the number of energy groups. For this purpose, three numerical methods based on the Bondarenko model are devised. We refer to this new method as the advanced Bondarenko method. With this advanced Bondarenko method, accuracy improvement can be realized without any significant increase in computational time and memory storage.

The present paper is organized as follows; Sections 2 and 3 describe fundamental theory of the advanced Bondarenko method and actual multi-group library generation based on this method. Section 4 is devoted to investigate performance of multi-group calculations with a multi-group library based on the advanced Bondarenko method. Finally, conclusion of the present study is provided in Section 5 .

\section{Theory of the advanced Bondarenko method}

There are three specific numerical methods in the advanced Bondarenko method; Bell factor optimization, application and extension of resonance interference factors, and correction factors for current-weighted total cross sections. Fundamental theory of them is described in this section.

\subsection{Bell factor optimization}

The correction factor of Bell, the Bell factor, was proposed to improve the one-term rational approximation for neutron escape probability from a fuel region, $P_{e s c}(E)$, as follows:

$$
P_{e s c}(E) \approx \frac{a}{\bar{l} \Sigma_{t}^{F}(E)+a}
$$

where $a$ is the Bell factor, $\bar{l}$ is the mean chord length of the fuel region and $\Sigma_{t}^{F}(E)$ is the total macroscopic cross section of the fuel region at neutron incident energy $E$. It is mentioned in the textbook by Stamm'ler and Abbate that a unique value of 1.16 is the best choice for both rod and slab lattices(Stamm'ler and Abbate, 1983) whereas optimum values of $a$ depend on the neutron energy $E$. In the resonance selfshielding calculations, the most important is to calculate energy-averaged cross sections accurately. Thus 
Let us consider an isolated fuel pin cell consisting of a fuel region and a moderator region. Neutron flux energy spectrum in the fuel region, $\phi^{F}(E)$, can be represented by the following equation based on the narrow resonance approximation to scattering sources(Stamm'ler and Abbate, 1983):

$$
\phi^{F}(E)=\frac{\Sigma_{0}^{F}}{\Sigma_{t}^{F}(E)}\left(1-P_{e s c}(E)\right)+P_{e s c}(E),
$$

where $\Sigma_{0}^{F}$ is the potential scattering cross section of the fuel region. If the one-term rational approximation is introduced to the fuel escape probability representation, the neutron flux energy spectrum can be approximately written as follows:

$$
\tilde{\phi}^{F}(E)=\frac{\bar{l} \Sigma_{0}^{F}+a}{\bar{l} \Sigma_{t}^{F}(E)+a} \propto \frac{1}{\left(N_{i} \sigma_{t}^{i}(E)+\sum_{j \neq i} N_{j} \sigma_{t}^{j}\right)+a / \bar{l}} \propto \frac{1}{\sigma_{t}^{i}(E)+\sigma_{0}+a /\left(N_{i} \bar{l}\right)},
$$

where $N_{i}$ and $\sigma_{t}^{i}(E)$ are number density and microscopic total cross section of nuclide $i$, and $\sigma_{0}$ is the background cross section without heterogeneous correction. The background cross section is defined as

$$
\sigma_{0}=\frac{\sum_{j \neq i} N_{j} \sigma_{t}^{j}}{N_{i}} .
$$

Note that nuclides except the nuclide $i$ are considered non-resonant. Optimum Bell factor which reproduces energy-averaged cross section of group $g$ can be defined to preserve the following equation:

$$
\begin{aligned}
\frac{\int_{E \in g} \sigma_{x}^{i}(E) \phi^{F}(E) d E}{\int_{E \in g} \phi^{F}(E) d E} & =\frac{\int_{E \in g} \sigma_{x}^{i}(E) \tilde{\phi}^{F}(E) d E}{\int_{E \in g} \tilde{\phi}^{F}(E) d E}, \\
\frac{\int_{E \in g} \sigma_{x}^{i}(E)\left\{\frac{\Sigma_{0}^{F}}{\Sigma_{t}^{F}(E)}\left(1-P_{e s c}(E)\right)+P_{e s c}(E)\right\} d E}{\int_{E \in g}\left\{\frac{\Sigma_{0}^{F}}{\sum_{t}^{F}(E)}\left(1-P_{e s c}(E)\right)+P_{e s c}(E)\right\} d E} & =\frac{\int_{E \in g} \sigma_{x}^{i}(E)\left\{\frac{1}{\sigma_{t}^{i}(E)+\sigma_{0}+a /\left(N_{i} \bar{l}\right)}\right\} d E}{1},
\end{aligned}
$$

where $\sigma_{x}^{i}(E)$ is the microscopic cross section of nuclide $i$ and reaction type $x$. If the left hand side of Eq. (5) can be calculated, nuclide- and energy group-wise optimum Bell factors can be determined from this equation. The nuclide- and energy group-wise optimum Bell factors should be dependent on reaction type $x$, mean chord length $\bar{l}$, potential scattering cross section $\Sigma_{0}^{F}$ and background cross section $\sigma_{0}$. If the optimum Bell factors significantly depend on system-dependent parameters such as $\bar{l}$ and $\sigma_{0}$, these should be calculated for each calculated system. However, if this dependence is not so significant, unique values which are calculated from a one representative system can be commonly used for other systems. This will be discussed later in the present paper. 


\subsection{Application and extension of resonance interference factors}

It has been well recognized in the field of reactor physics that the resonance interference effect among different resonant nuclides is quite important. While there are several ways to consider this effect, the resonance interference factor (R-factor) method was proposed in the frame of the Bondarenko method(Takano, 1980). The R-factor is one additional parameter in a self-shielding factor table; the R-factor is defined as a number density ratio of two resonant nuclides, which are a resonant nuclide in consideration and another target resonant nuclide with which the resonance interference effect is considered. Self-shielding factors are pretabulated at several different values of R-factors.

In the original R-factor method, the target nuclide is prefixed: uranium-238 is a target nuclide for all the resonant nuclides except uranium-238 itself, and plutonium-239 is a target nuclide for uranium-238. In the present study, we extend the R-factor method such that the target nuclide with which resonance interference effect is considered is dependent on nuclide and energy group. Furthermore, for cases where the resonance interference effects with several different nuclides are important, we newly devise the following multiple resonance interference treatment method. In such a case, the energy-averaged cross section of group $g, \sigma_{g}$, is calculated as

$$
\sigma_{g}=\left(\prod_{j=1}^{J} \frac{\sigma_{g}^{j}}{\sigma_{g}^{w / o R I}}\right) \cdot \sigma_{g}^{w / o R I}
$$

where $\sigma_{g}^{j}$ is a tabulated cross section in which the resonance interference with the nuclide $j$ as a target is considered and $\sigma_{g}^{w / o R I}$ is one in which no resonance interference is considered. This method is based on an assumption that resonance interference effect between a pair of resonant nuclides is independent on that between a different pair of resonant nuclides.

\subsection{Corrections for current-weighted total cross section}

It has been reported that a coarse-energy group calculation does not reproduce neutron multiplication factor of a reference fine-energy group calculation even if fine-group neutron flux obtained from the reference calculation is used as a weight function in cross section group collapsing(Sugimura and Yamamoto, 2007). This collapsing error in a coarse group calculation is caused by a fact that fine-group total cross sections are collapsed using scalar neutron flux as a weight function(Takeda and Kitada, 2002).

The collision term of the one-dimensional neutron transport equation in the continuous energy representation is written as $\Sigma_{t}(x, E) \psi(x, E, \mu)$, where $\psi(x, E, \mu)$ is angular neutron flux directed to $\cos ^{-1} \mu$ at spatial position $x$ and energy $E$. Since energy-averaged cross sections are defined so as to reproduce energyintegrated reaction rate, energy-averaged total cross sections should be dependent on $\mu$. This treatment, however, makes multi-group neutron transport equation much more complicated, so the angular neutron flux is expanded by the Legendre polynomials and Legendre moment-dependent multi-group total cross sections 
are defined. In this case, the collision term in the multi-group representation is written as

$$
\sum_{l} \frac{2 l+1}{2} P_{l}(\mu) \Sigma_{t, g}^{l}(x) \phi_{g}^{l}(x)
$$

where $P_{l}(\mu)$ is the $l$ th Legendre polynomial and $\phi_{g}^{l}$ is the $l$ th Legendre moment of the $g$ th group angular neutron flux. The Legendre moment-dependent multi-group cross sections are defined as

$$
\Sigma_{t, g}^{l}(x)=\frac{\int_{E \in g} \Sigma_{t}(x, E) \phi^{l}(x, E) d}{\int_{E \in g} \phi^{l}(x, E) d E}
$$

where $\phi^{l}(x, E)$ is the $l$ th Legendre moment of $\psi(x, E, \mu)$. This representation for the multi-group collision term shown in Eq. (7) is not suited to the usual numerical solvers for neutron transport, so this is modified to

$$
\begin{aligned}
\sum_{l} \frac{2 l+1}{2} P_{l}(\mu) \tilde{\Sigma}_{t, g}(x) \phi_{g}^{l}(x)-\sum_{l} \frac{2 l+1}{2} P_{l}(\mu) & \left(\tilde{\Sigma}_{t, g}(x)-\Sigma_{t, g}^{l}(x)\right) \\
= & \tilde{\Sigma}_{t, g}(x) \psi_{g}(x, \mu)-\sum_{l} \frac{2 l+1}{2} P_{l}(\mu)\left(\tilde{\Sigma}_{t, g}(x)-\Sigma_{t, g}^{l}(x)\right),
\end{aligned}
$$

where $\tilde{\Sigma}_{t, g}$ is the collision cross section and can be arbitrarily chosen. The first term of the right hand side of Eq. (9) is the collision term and the second term is treated in the scattering source term; the $g$ th group self-scattering cross section of the $l$ th Legendre moment is modified to

$$
\hat{\Sigma}_{g \rightarrow g}^{l}(x)=\Sigma_{g \rightarrow g}^{l}(x)+\tilde{\Sigma}_{t, g}(x)-\Sigma_{t, g}^{l}(x),
$$

where $\hat{\Sigma}_{g \rightarrow g}^{l}(x)$ and $\Sigma_{g \rightarrow g}^{l}(x)$ are corrected and original self-scattering cross sections of the $l$ th Legendre moment. If $\Sigma_{t, g}^{0}(x)$ is used as the collision cross section $\tilde{\Sigma}_{t, g}(x)$, the transport cross section $\Sigma_{t r, g}(x)$, which is frequently used in neutron transport calculations, is defined as

$\Sigma_{t r, g}(x)=\Sigma_{t, g}^{0}(x)-\sum_{g^{\prime}} \hat{\Sigma}_{g \rightarrow g^{\prime}}^{1}(x)=\Sigma_{t, g}^{0}(x)-\left(\sum_{g^{\prime}} \Sigma_{g \rightarrow g^{\prime}}^{1}(x)+\Sigma_{t, g}^{0}(x)-\Sigma_{t, g}^{1}(x)\right)=\Sigma_{t, g}^{1}(x)-\sum_{g^{\prime}} \Sigma_{g \rightarrow g^{\prime}}^{1}(x)$.

This suggests that the first Legendre moment-weighted (current-weighted) total cross section should be used in the transport cross section definition. In the advanced Bondarenko method, we propose to use correction factors to calculate current-weighted total cross sections.

Neutron flux energy spectrum of a fuel region in a heterogeneous lattice system can be well approximated by the same representation as the homogeneous system in the equivalence principle, but there has been no theoretical representations for neutron current energy spectrum in a heterogeneous system. In some cases such as fast neutron reactors analyses, neutron current is approximated by the following equation:

$$
\phi^{1}(E) \propto \frac{1}{\left\{\Sigma_{t}(E)\right\}^{2}},
$$


which is derived from the homogeneous $\mathrm{P}_{1}$ theory. It is hardly to say that this is applicable to a heterogeneous system of light water reactor fuel pin cell. Another way is to assume that current-weighted total cross sections are the same as flux-weighted total cross sections, but there is no guarantee to assure its accuracy. In the advanced Bondarenko method, we directly evaluate current-weighted total cross sections from fine-energy group calculations and implement them to a coarse-energy group library.

\section{Generation of multi-group cross section library based on the advanced Bondarenko method}

A deterministic reactor physics code system CBZ, which is being developed at Hokkaido University, has its own multi-group cross section library named CBZLIB. CBZLIB is generated by two evaluated nuclear data file processing codes, NJOY-99(MacFarlane and Muir, 1994) and TIMS-1(Takano, 1980), and other utility programs. The number of energy groups and the original evaluated nuclear data files can be arbitrarily chosen for users' application purposes. For thermal neutron reactors applications, 107-energy group structure which was originated in the SRAC code(Okumura, 2007) is generally used. In the SRAC 107-energy group structure, equal lethargy width of 0.25 is assigned to every energy group above $1.85540 \mathrm{eV}$. The highest energy is $20 \mathrm{MeV}$, so 62 energy groups are structured above $1.85540 \mathrm{eV}$. From $0.41399 \mathrm{eV}$ to $1.85540 \mathrm{eV}$, constant lethargy width of 0.125 is assigned, and below $0.41399 \mathrm{eV}$ lethargy width of energy groups are different from each other. Upper energy boundaries of some energy groups in the resonance range are shown in Table 1.

In the present study, we generate a 107-group CBZLIB from the evaluated nuclear data file JENDL4.0(Shibata, 2011). Infinite dilution cross sections, scattering matrices including thermal scattering up to the fifth Legendre moment and self-shielding factor tables are generated by NJOY-99. For important actinoid nuclides, uranium-235, -238, plutonium-238, -239, -240, -241, -242 and americium-241, self-shielding factors depending on R-factors are re-calculated by TIMS-1 and updated. The TIMS-1 code solves the ultrafine energy group slowing-down equation for a homogeneous system consisting of a resonant nuclide, a moderator material which is hydrogen in the present case, and another resonant nuclide with which the resonance interference effect is considered.

In order to generate CBZLIB based on the advanced Bondarenko method for light water reactor (LWR) applications, we prepare pressurized water reactor (PWR) and boiling water reactor (BWR) fuel pin cell models. In all the PWR cells, pin pitch, pellet outer radius, cladding outer radius are $1.265 \mathrm{~cm}, 0.412 \mathrm{~cm}$ and $0.476 \mathrm{~cm}$, and temperatures of fuel, cladding and moderator regions are $968.8 \mathrm{~K}, 604.0 \mathrm{~K}$ and $574.2 \mathrm{~K}$. Fuel compositions of eight PWR cells are shown in Table 2. On the BWR cells, geometrical specifications are dependent on step. In the step I and step II cells, pin pitch, pellet outer radius, cladding outer radius are $1.63 \mathrm{~cm}, 0.529 \mathrm{~cm}$ and $0.615 \mathrm{~cm}$, respectively. In the step III cells, those are $1.44 \mathrm{~cm}, 0.490 \mathrm{~cm}$ and $0.560 \mathrm{~cm}$. Temperatures of fuel, cladding and moderator regions are $968.8 \mathrm{~K}, 559.0 \mathrm{~K}$ and $559.0 \mathrm{~K}$ for the 
BWR cells. Fuel compositions of sixteen BWR cells are shown in Table 3 . In the following, we refer to the PWR(BWR) cell whose ID is $n$ as PWR- $n(\mathrm{BWR}-n)$.

\subsection{Calculations of optimum Bell factors}

As described in the previous section, optimum Bell factors are determined so as to preserve Eq. (5). In the present study, energy integration is conducted numerically with a probability table, which is generated by the MOMENTOF code(Chiba and Unesaki, 2006) based on Ribon's moment method(Ribon, 1986). The original nuclear data file of the probability table is JENDL-4.0. Potential scattering cross sections of heavy nuclides and oxygen in the fuel region are assumed 10 barn and 4 barn, respectively, and the macroscopic potential scattering cross section in fuel region is assumed $0.4 \mathrm{~cm}^{-1}$.

Optimum Bell factors for uranium-238 are calculated for the PWR-2 cell so as to preserve energyaveraged capture cross sections. To observe optimum Bell factors dependence on system parameters, such as temperature, chord length, number density and dilution cross sections, optimum Bell factors are calculated in different conditions in which one of these system parameters is multiplied by a factor of 1.3. The results are shown in Fig. 1. Note that default values of 1.2 for Bell factors are assigned to some energy groups in which probability tables are not successfully produced. These results suggest that optimum Bell factors are not significantly sensitive to changes in system parameters. Next optimum Bell factors for uranium-235 are shown in Fig. 2. These are calculated for the PWR-2 cell, and energy-averaged fission and capture cross sections are preserved. We can see that choice of the target reaction type is not important. The above results suggest that optimum Bell factors can be predefined for a reference nuclear system and those can be applicable to other systems whose characteristics do not change from the reference system significantly.

Based on this concept, optimum Bell factors for LWR applications are calculated. Those for uranium-235 and -238 are calculated for the PWR-2 cell and those for plutonium-239, -240, -241 and -242 are calculated for the PWR-6 cell. Background cross sections without heterogeneous corrections, which are used in the Bell factor optimization, are shown in Fig. 3. The obtained optimum Bell factors, which are implemented to CBZLIB, are shown in Fig. 4. It is interesting to point out that the optimum Bell factors for uranium-238 take relatively small values in energy groups where wide resonance peaks exist. This makes background cross sections for uranium-238 small and promotes the resonance self-shielding effect, so it is expected that energy-averaged uranium-238 capture cross sections become small in these energy groups.

\subsection{Resonance interference factor setting}

As described above, in the advanced Bondarenko method, target nuclides, with which the resonance interference effect is taken into account, are dependent on energy groups, and multiple resonance interference effects can be approximately considered. While it is possible to consider the resonance interference effects 
between all the possible pairs of resonant nuclides by this multiple resonance interference treatment, only important resonance interference is treated here to avoid increase of computational burden.

The resonance interference factors are introduced to the following important heavy nuclides; uranium-235, -238, plutonium-239, -240, -241, -242 and americium-241. Target nuclides with which resonance interference is taken into account are listed in Table $\mathbf{4}$ for these nuclides. The multiple resonance interference is considered in the energy groups 48, 50 and 53.

\subsection{Calculations of correction factors for current-weighted total cross sections}

As described in the preceding section, current-weighted $(\mathrm{CW})$ total cross sections are evaluated directly from fine-energy group calculations in our procedure. For this purpose, we prepare a 707-energy group library from JENDL-4.0 by NJOY-99. The energy group structure of this 707-group library is prepared so that energy groups from $1.8554 \mathrm{eV}$ to $961.12 \mathrm{eV}$ in the SRAC 107-group structure are divided into 25 equal lethargy width energy groups. Thermal scattering data are not prepared for this 707-group library.

With this 707-group library, eigenvalue calculations are carried out for all the PWR and BWR fuel cells being considered. In order to calculate angular neutron flux, a MEC module of CBZ, which is based on the method of characteristics, is employed as a neutron transport solver. The fuel cell is spatially discretized as shown in Fig. 5. Reference infinite neutron multiplication factors $k_{\infty}$, neutron flux energy spectra and neutron current energy spectra along the $x$-direction at one spatial mesh in the fuel region are obtained with the 707-group calculations, and group collapsing of 707-group total cross sections to the SRAC-107 group structure is conducted with the neutron flux and the neutron current. With this 107-group cross section, coarse-group calculations are carried out by MEC for all the cells and $k_{\infty}$ are obtained. Reproduction errors of $k_{\infty}$ are shown in Fig. 6. In this figure, results denoted as "Current-weighted" are obtained with the CW total cross sections and results denoted as "Flux-weighted" are obtained with the flux-weighted (FW) total cross sections as CW cross sections. These results show that use of CW cross sections significantly reduce errors induced by energy group collapsing.

Numerical results suggest that treatment for uranium-238 microscopic total cross sections are important. Averaged ratios of uranium-238 CW microscopic total cross sections to FW cross sections over all the PWR and BWR cells are shown in Fig. 7. In this figure, possible ranges of the cross section ratios are also shown by error bars. We can see that the CW total cross sections are larger than the FW cross sections in some energy groups where wide resonances exist, and the ratios of the CW total cross sections to the FW total cross sections do not significantly dependent on calculated systems. In the advanced Bondarenko method, these averaged ratios are implemented as correction factors to deduce CW total cross sections for the uranium-238 resonance ranges. In addition to uranium-238, a ratio of 3.15 is also implemented to the 61th energy group of plutonium-242 to reduce cross section collapsing errors in MOX cell calculations. 


\section{Performance of the advanced Bondarenko method}

In order to quantify accuracy of multi-group calculations with CBZLIB based on the advanced Bondarenko method, $k_{\infty}$ and energy-averaged cross sections of the LWR fuel cells being considered are calculated with CBZ. Reference solutions are obtained with the continuous-energy Monte Carlo code MVP-II(Nagaya, 2005).

Background cross sections in heterogeneous fuel cells are evaluated by the Dancoff factor method. Dancoff factors are calculated for each energy group without any geometrical simplification with the perfect reflective boundary conditions. The concept of the wide resonance approximation is introduced, so scattering cross sections of actinoid nuclides are not taken into account in background cross sections evaluations below $40 \mathrm{eV}$. The Bondarenko iterations are conducted two times for each energy group. Energy-averaged 107-group cross sections are calculated with the advanced Bondarenko method described in the preceding sections.

With the obtained cross sections, 107-group eigenvalue calculations are performed with a collision probability module PJI, and $k_{\infty}$ and energy-averaged cross sections are obtained. Scattering anisotropy is taken into account by the transport approximation.

\subsection{Infinite neutron multiplication factors}

Reproduction errors of $k_{\infty}$ are shown in Fig. 8. Although underestimation of about $0.1 \% \Delta k / k k^{\prime}$ is systematically observed, good agreements within $0.15 \% \Delta k / k k^{\prime}$ differences are obtained with the advanced Bondarenko method. No significant biases on fuel compositions, geometrical specifications and void ratios are observed. In order to see contribution of each numerical method of the advanced Bondarenko method to the accuracy improvement, other calculations with different numerical conditions are performed. Reproduction errors of $k_{\infty}$ with several conditions are shown in Fig. 9. Each of numerical methods, the optimum Bell factors, the extended resonance interference factors and the CW total cross section corrections, contributes to the accuracy improvement, and the last one is the most dominant. Note that there is no significant difference in computation time among these different calculation options.

\subsection{Energy-averaged cross sections}

First, reproduction errors of energy-averaged uranium-238 capture cross sections are shown in Fig. 10. In order to grasp significance of errors in this cross section to $k_{\infty}$, relative sensitivities of $k_{\infty}$ with respect to this cross section are shown in Fig. 11. Large errors over $5 \%$ are observed in the energy group 42 (275.4 to $353.6 \mathrm{eV}$ ). It might come from neglecting the resonance interference effect between uranium-238 and zirconium-91 included in the clad region. This error is not significant because sensitivity of $k_{\infty}$ in this energy group is relatively small in comparison with those in other energy groups as shown in Fig. 11. Except for this energy group, energy-averaged uranium-238 capture cross sections are reproduced within $6 \%$ differences to the reference Monte Carlo solutions. 
Next, reproduction errors of energy-averaged uranium-235 and plutonium-239 fission cross sections are shown in Figs. 12 and 13. Energy-averaged fission cross sections are reproduced within 1.5\% differences in uranium-235 and 3\% differences in plutonium-239 to the reference Monte Carlo solutions.

\section{Concluding remarks}

The advanced Bondarenko method for resonance self-shielding calculations has been devised and proposed. This method is based on three numerical methods; the Bell factor optimization for accurate fuel escape probability representation, extension of resonance interference factors and correction factors for current-weighted total cross sections. A 107-group library for light water reactor applications based on the advanced Bondarenko method has been generated for a reactor physics code system CBZ. Performance of the CBZ code with this 107-group library has been examined against a suit of light water reactor cell problems. The infinite neutron multiplication factors calculated with CBZ have agreed with reference continuous-energy Monte Carlo solutions within $0.15 \% \Delta k / k k^{\prime}$ differences, and no significant biases on fuel compositions and geometrical specifications have been observed. Energy-averaged cross sections of uranium238 capture, uranium-235 fission and plutonium-239 fission reactions have been also examined. Numerical tests have revealed that significant accuracy improvements in the resonance self-shielding calculations are realized by adopting the advanced Bondarenko method without any significant increase of computational burden.

\section{Acknowledgements}

Part of this work was financially supported by the nuclear regulatory authority of Japan. The authors are grateful to Mr. Kenta Matsuura of Hokkaido University for his support to this study.

\section{References}

Bondarenko, I.I., 1964. Group constants for nuclear reactor calculations, Consultants Bureau, New York.

Chiba, G., Unesaki, H., 2006. Improvement of moment-based probability table for resonance self-shielding calculation, Ann. Nucl. Energy, 33. 1141-1146.

MacFarlane, R.E., Muir, D.W., 1994. The NJOY nuclear data processing system, version 91, LA-12740-M, Los Alamos National Laboratory.

Nagaya, Y., Okumura, K., Mori, T., Nakagawa, M., 2005. MVP/GMVP II: The general purpose Monte Carlo codes for neutron and photon transport calculations based on continuous energy and multigroup methods, JAERI 1348, Japan Atomic Energy Research Institute.

Okumura, K., Kugo, T., Kaneko, K., Tsuchihashi, K., 2007. SRAC2006: a comprehensive neutronics calculation code system, JAEA-Data/Code 2007-004, Japan Atomic Energy Agency. 
Ribon, P., 1986. Probability tables and gauss quadrature application to neutron cross-sections in the unresolved energy range, Proc. of topical meeting on advanced in reactor physics and safety, September 17-19, 1986, Vol. 1, 280, Saratoga Springs, USA.

Rhodes, J., Gheorghiu, N., Ferrer, R., 2012. CASMO5 JENDL-4.0 and ENDF/B-VII.1beta4 libraries, Proc. of international meeting on reactor physics, PHYSOR2012, April 15-20, 2012, Knoxville, USA.

Sanchez, R., Zmijarevic, I., Coste-Delclaux, M., Masiello, E., Santandrea, S., Martinollf, E., Villate, L., Schwartz, N., Guler, N., 2010. APOLLO2 year 2010, Nucl. Eng. Technol., 42, 474-499.

Shibata, K., Iwamoto, O., Nakagawa, T., Iwamoto, N., Ichihara, A., Kunieda, S., Chiba, S., Furutaka, K., Otuka, N., Ohsawa, T., Murata, T., Matsunobu, H., Zukeran, A., Kamada, S., Katakura, J., 2011. JENDL-4.0: a new library for nuclear science and engineering, J. Nucl. Sci. Technol., 48, 1-30.

Stamm'ler, R.J.J., Abbate, M.J., 1983. Methods of steady-state reactor physics in nuclear design, Academic Press, London.

Sugimura, N., Yamamoto, A., 2007. Resonance treatment based on ultra-fine-group spectrum calculation in the AEGIS code, J. Nucl. Sci. Technol., 44, 958-966.

Takano, H., 1980. TIMS-1: a processing code for production of group constants of heavy resonant nuclei, JAERI 1267, Japan Atomic Energy Research Institute.

Takeda, T., Kitada, T., 2002. Direction and region dependent cross sections for use to MOX fuel analysis, J. Nucl. Sci. Technol., Supp. 2, 1057-1060. 
Table 1: Upper energy boundaries of SRAC 107-energy group structure (unit: eV)

\begin{tabular}{cccccccc}
\hline Group & Boundary & Group & Boundary & Group & Boundary & Group & Boundary \\
\hline 36 & 1584.6 & 37 & 1234.1 & 38 & 961.12 & 39 & 748.52 \\
40 & 582.95 & 41 & 454.00 & 42 & 353.58 & 43 & 275.37 \\
44 & 214.45 & 45 & 167.02 & 46 & 130.07 & 47 & 101.30 \\
48 & 78.893 & 49 & 61.442 & 50 & 47.851 & 51 & 37.267 \\
52 & 29.023 & 53 & 22.603 & 54 & 17.604 & 55 & 13.710 \\
56 & 10.677 & 57 & 8.3153 & 58 & 6.4759 & 59 & 5.0435 \\
60 & 3.9279 & 61 & 3.0590 & 62 & 2.3824 & 63 & 1.8554 \\
\hline
\end{tabular}


Table 2: Compositions of PWR fuels

\begin{tabular}{ccccc}
\hline ID & $\begin{array}{c}\text { Fuel } \\
\text { type }\end{array}$ & $\begin{array}{c}\text { U-235 } \\
\text { enrichment [\%] }\end{array}$ & $\begin{array}{c}\mathrm{Pu} \\
\text { enrichment }[\%]\end{array}$ & $\begin{array}{c}\text { Pu isotopic weight ratio [\%] } \\
\left({ }^{238} \mathrm{Pu} /{ }^{239} \mathrm{Pu} /{ }^{240} \mathrm{Pu} /{ }^{241} \mathrm{Pu} /{ }^{242} \mathrm{Pu} /{ }^{241} \mathrm{Am}\right)\end{array}$ \\
\hline 1 & $\mathrm{UO}_{2}$ & 3.4 & & \\
2 & $\mathrm{UO}_{2}$ & 4.1 & & \\
3 & $\mathrm{UO}_{2}$ & 4.7 & & \\
4 & MOX & 0.2 & 13 & $4.1 / 45.4 / 25.3 / 9.6 / 13.0 / 2.6$ \\
5 & MOX & 0.2 & 5 & $2.1 / 54.5 / 25.0 / 9.3 / 6.4 / 2.7$ \\
6 & MOX & 0.2 & 10 & $2.1 / 54.5 / 25.0 / 9.3 / 6.4 / 2.7$ \\
7 & MOX & 0.2 & 13 & $2.1 / 54.5 / 25.0 / 9.3 / 6.4 / 2.7$ \\
8 & MOX & 0.2 & 5 & $0.04 / 79.24 / 17.76 / 2.36 / 0.36 / 0.24$ \\
\hline
\end{tabular}


Table 3: Compositions of BWR fuels

\begin{tabular}{|c|c|c|c|c|c|c|}
\hline ID & $\begin{array}{l}\text { Fuel } \\
\text { type }\end{array}$ & Step & $\begin{array}{c}\text { Void } \\
\text { ratio } \\
{[\%]}\end{array}$ & $\begin{array}{l}\text { U-235 } \\
\text { enrichment } \\
{[\%]}\end{array}$ & $\begin{array}{c}\mathrm{Pu} \\
\text { enrichment } \\
{[\%]}\end{array}$ & $\begin{array}{c}\mathrm{Pu} \text { isotopic weight ratio } \\
{[\%]} \\
\left({ }^{238} \mathrm{Pu} /{ }^{239} \mathrm{Pu} /{ }^{240} \mathrm{Pu} /{ }^{241} \mathrm{Pu} /{ }^{242} \mathrm{Pu} /{ }^{241} \mathrm{Am}\right)\end{array}$ \\
\hline 1 & $\mathrm{UO}_{2}$ & I & 0 & 3.0 & & \\
\hline 2 & $\mathrm{UO}_{2}$ & $\mathrm{I}$ & 40 & 3.0 & & \\
\hline 3 & $\mathrm{UO}_{2}$ & I & 70 & 3.0 & & \\
\hline 4 & $\mathrm{UO}_{2}$ & II & 0 & 3.8 & & \\
\hline 5 & $\mathrm{UO}_{2}$ & II & 40 & 3.8 & & \\
\hline 6 & $\mathrm{UO}_{2}$ & II & 70 & 3.8 & & \\
\hline 7 & $\mathrm{UO}_{2}$ & III & 0 & 4.1 & & \\
\hline 8 & $\mathrm{UO}_{2}$ & III & 40 & 4.1 & & \\
\hline 9 & $\mathrm{UO}_{2}$ & III & 70 & 4.1 & & \\
\hline 10 & MOX & II & 0 & 0.2 & 4 & $1.5 / 58.7 / 26.6 / 8.3 / 4.0 / 0.8$ \\
\hline 11 & MOX & II & 40 & 0.2 & 4 & $1.5 / 58.7 / 26.6 / 8.3 / 4.0 / 0.8$ \\
\hline 12 & MOX & II & 40 & 0.2 & 4 & $1.7 / 53.4 / 29.7 / 8.7 / 5.6 / 0.9$ \\
\hline 13 & MOX & II & 40 & 0.2 & 4 & $0.8 / 67.8 / 21.8 / 6.9 / 2.1 / 0.7$ \\
\hline 14 & MOX & II & 70 & 0.2 & 4 & $1.5 / 58.7 / 26.6 / 8.3 / 4.0 / 0.8$ \\
\hline 15 & MOX & II & 70 & 0.2 & 8 & $1.5 / 58.7 / 26.6 / 8.3 / 4.0 / 0.8$ \\
\hline 16 & MOX & II & 70 & 0.2 & 13 & $1.5 / 58.7 / 26.6 / 8.3 / 4.0 / 0.8$ \\
\hline
\end{tabular}


Table 4: Target nuclides with which resonance interference is taken into account

\begin{tabular}{|c|c|}
\hline Nuclide & Target nuclide (Energy group) \\
\hline Uranium-235 & Uranium-238 (All) \\
\hline \multirow[t]{3}{*}{ Uranium-238 } & Uranium-235 $(57,58)$ \\
\hline & Plutonium-240 $\left(48^{\dagger}, 50,51,53\right)$ \\
\hline & Plutonium-239 (Other groups, $48^{\dagger}$ ) \\
\hline \multirow[t]{4}{*}{ Plutonium-239 } & Plutonium-240 $\left(47,50^{\dagger}, 53^{\dagger}\right)$ \\
\hline & Plutonium-241 $\left(53^{\dagger}, 54,55,56\right)$ \\
\hline & Plutonium-242 (49) \\
\hline & Uranium-238 (Other groups, $50^{\dagger}, 53^{\dagger}$ ) \\
\hline \multirow[t]{2}{*}{ Plutonium-240 } & Plutonium-239 $(47,50)$ \\
\hline & Uranium-238 (Other groups) \\
\hline \multirow[t]{3}{*}{ Plutonium-241 } & Plutonium-239 $(52,54,55,56)$ \\
\hline & Americium-241 (59) \\
\hline & Uranium-238 (Other groups) \\
\hline \multirow[t]{3}{*}{ Plutonium-242 } & Plutonium-239 (49) \\
\hline & Americium-241 (61) \\
\hline & Uranium-238 (Other groups) \\
\hline \multirow[t]{2}{*}{ Americium-241 } & Plutonium-241 (59) \\
\hline & Uranium-238 (Other groups) \\
\hline
\end{tabular}

${ }^{\dagger}$ Multiple resonance interference factors are adopted. 
(a)

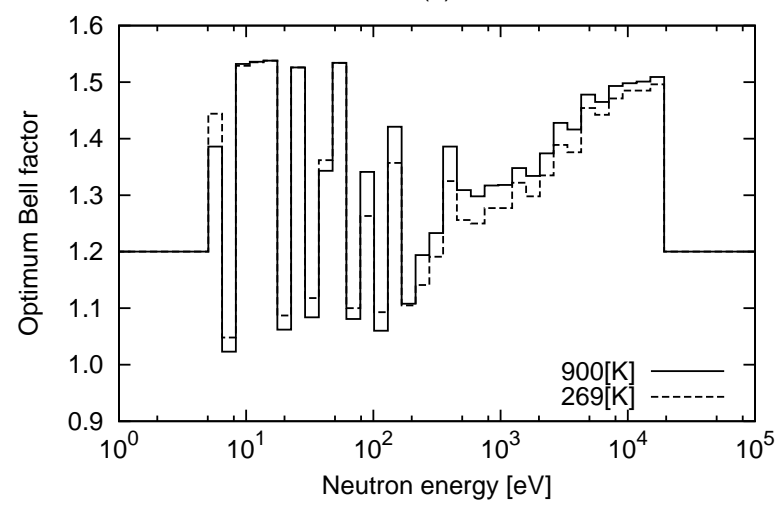

(b)

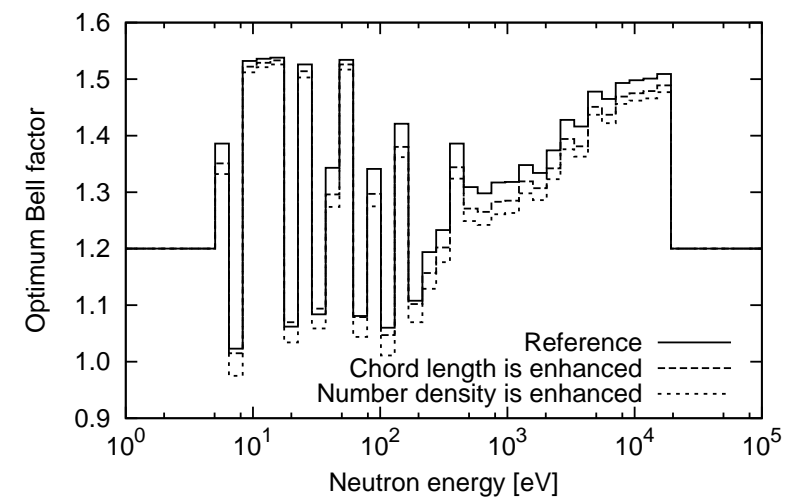

(c)

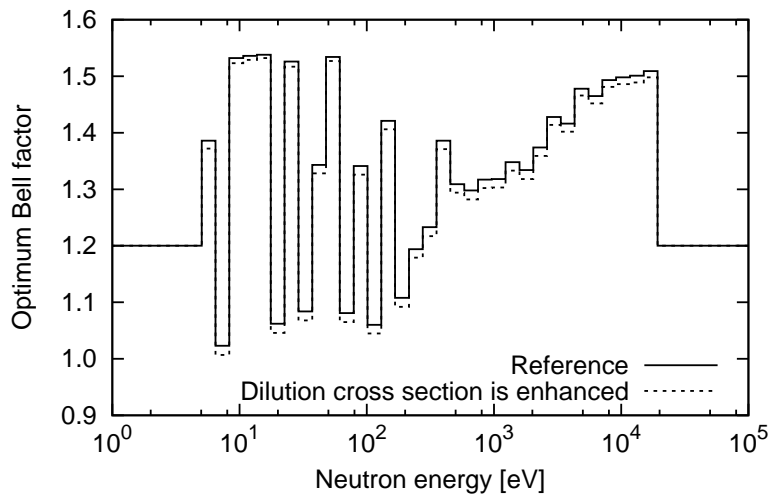

Figure 1: Optimum Bell factors for uranium-238 


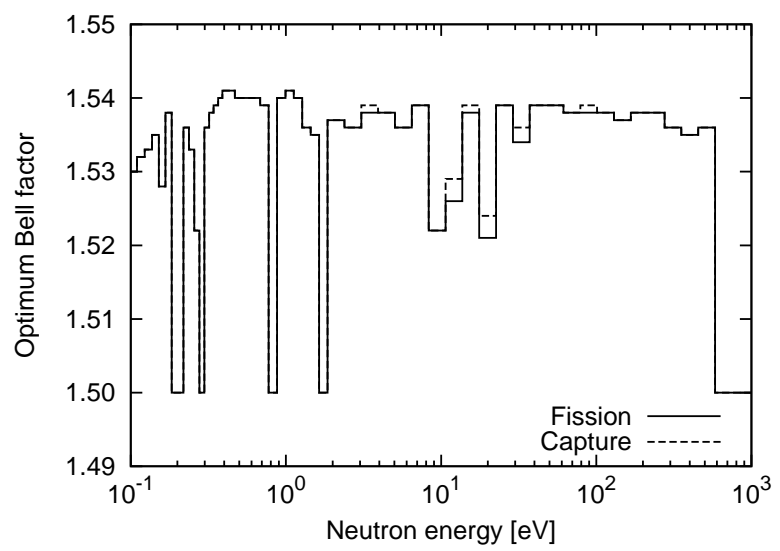

Figure 2: Optimum Bell factors for uranium-235 
(a) Uranium

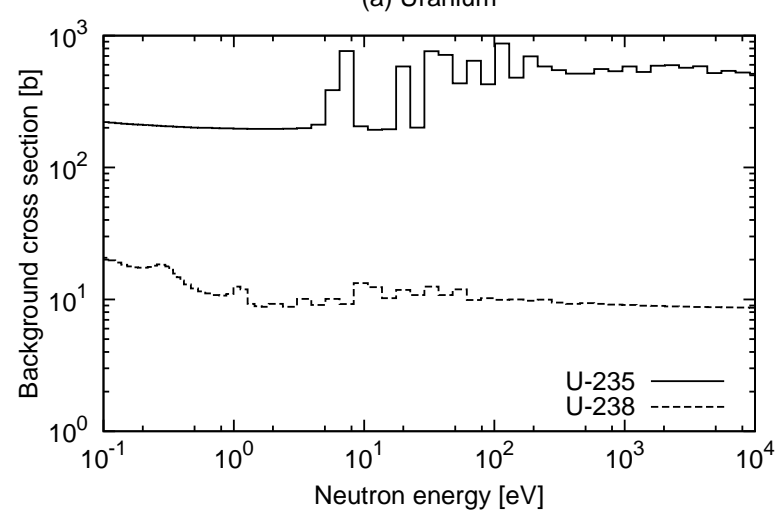

(b) Plutonium

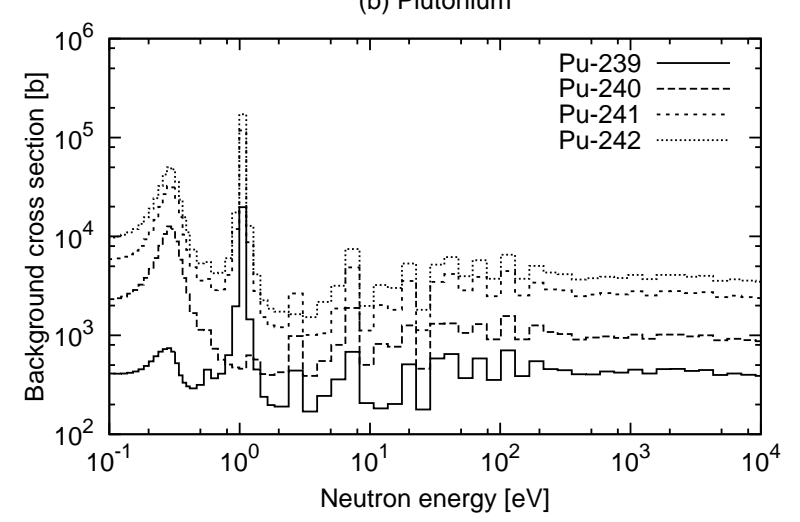

Figure 3: Background cross sections without heterogeneous correction 
(a) Uranium

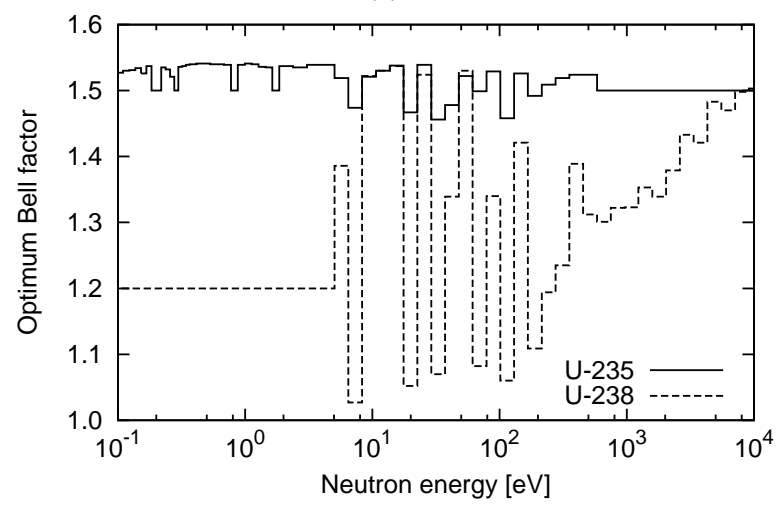

(b) Plutonium

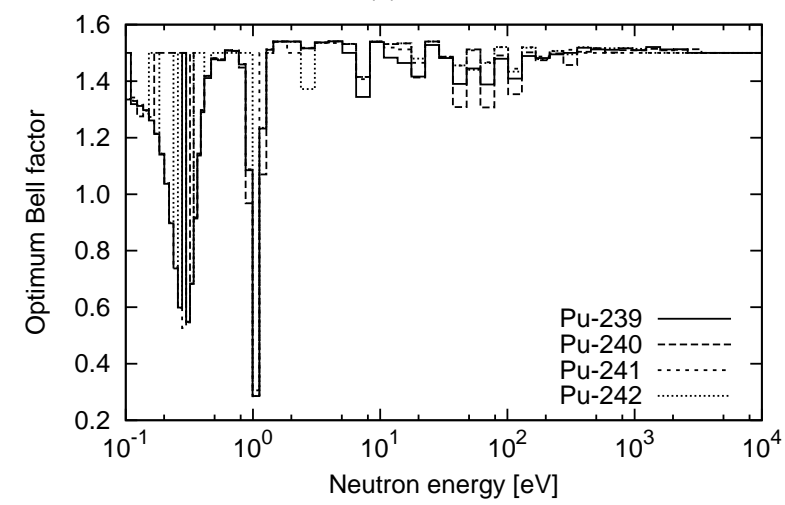

Figure 4: Optimum Bell factors implemented in CBZLIB 


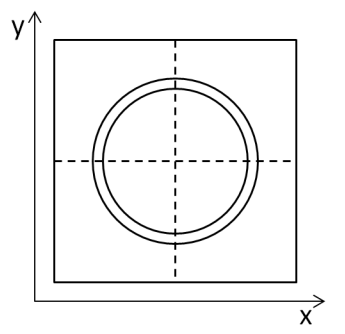

Figure 5: Spatial discretization of fuel cell in MEC 707-group calculation 
(a) PWR

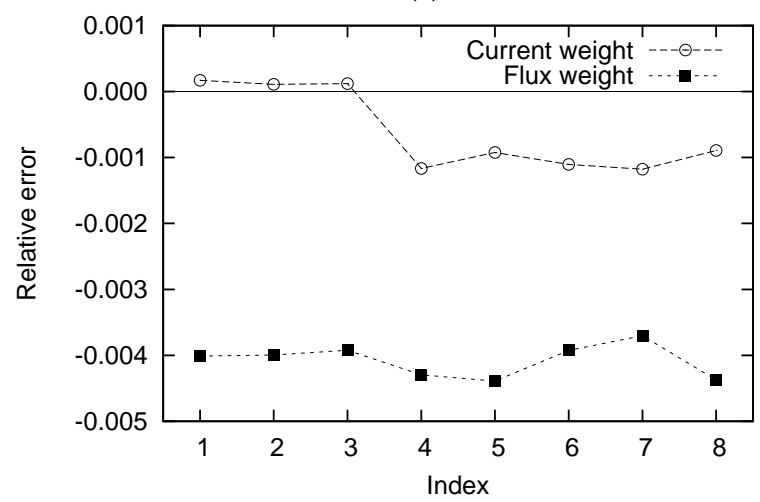

(b) BWR

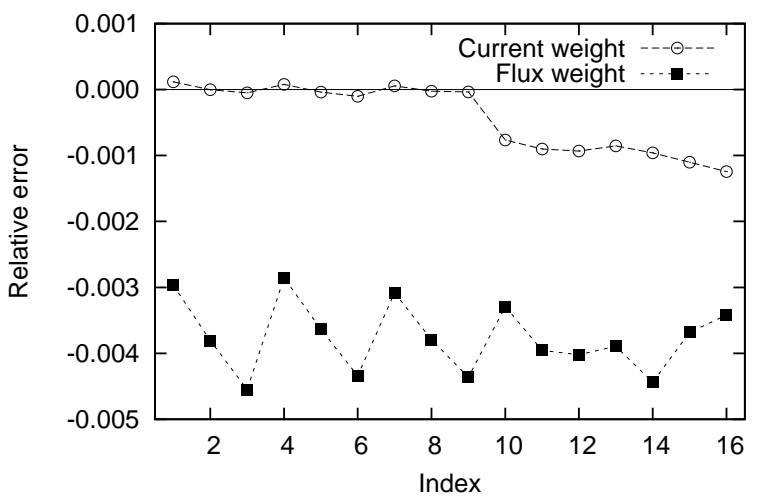

Figure 6: Reproduction errors of $k_{\infty}$ with different collapsing strategy for total cross section 


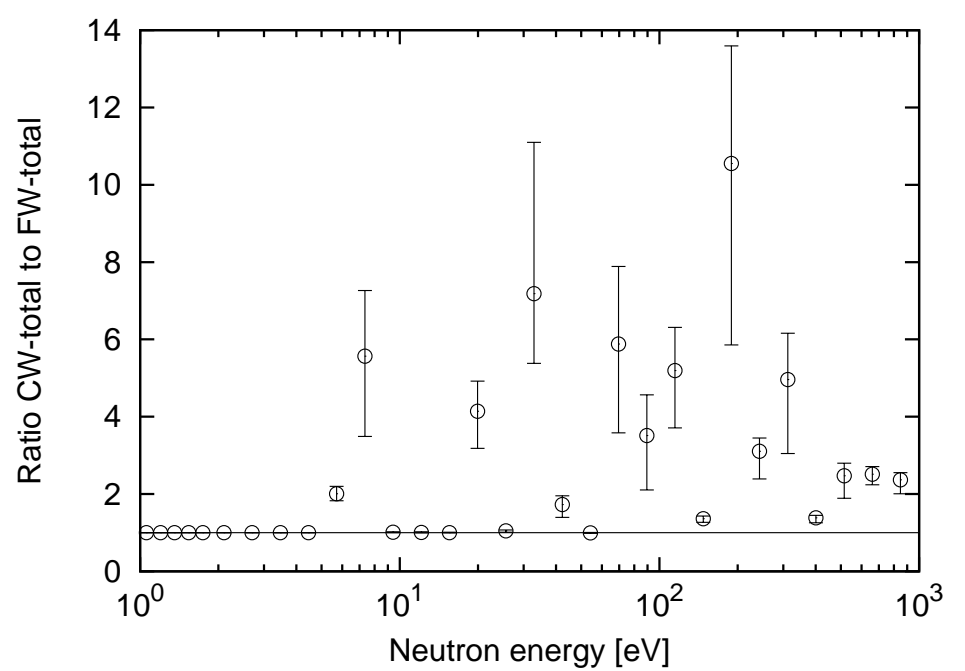

Figure 7: Ratios of current-weighted (CW) uranium-238 total cross sections to flux-weighted (FW) total cross sections 
(a) PWR

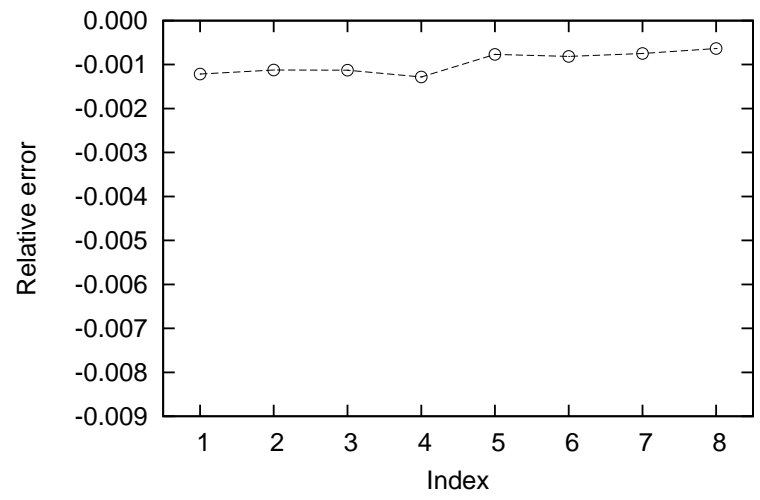

(b) BWR

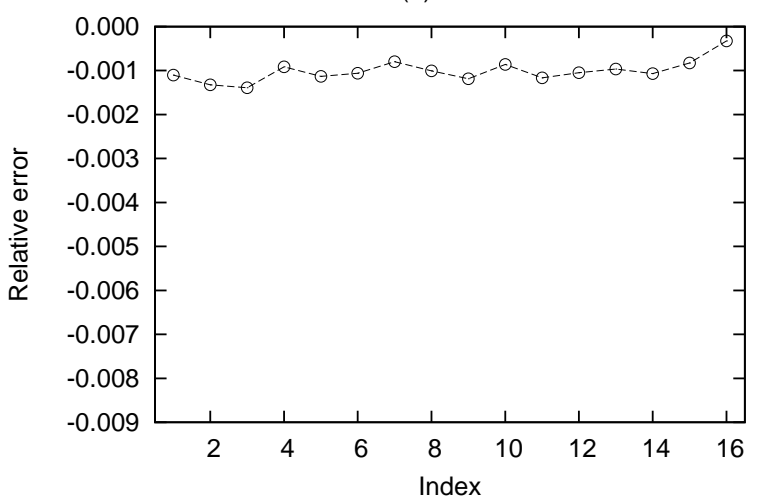

Figure 8: Reproduction errors in $k_{\infty}$ against Monte Carlo reference solutions 
(a) PWR

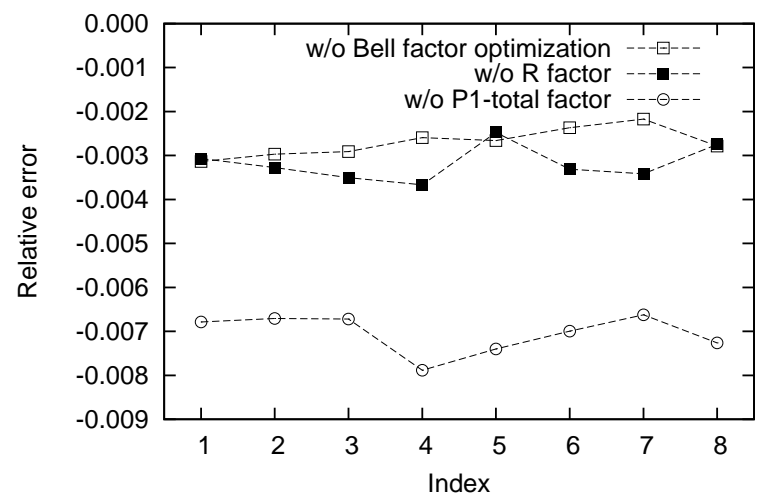

(b) BWR

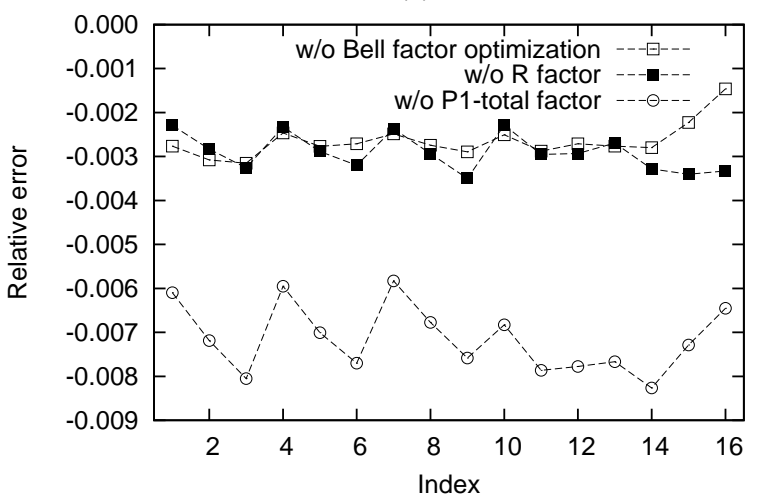

Figure 9: Reproduction errors in $k_{\infty}$ against Monte Carlo reference solutions with different conditions 
(a) PWR-UO2

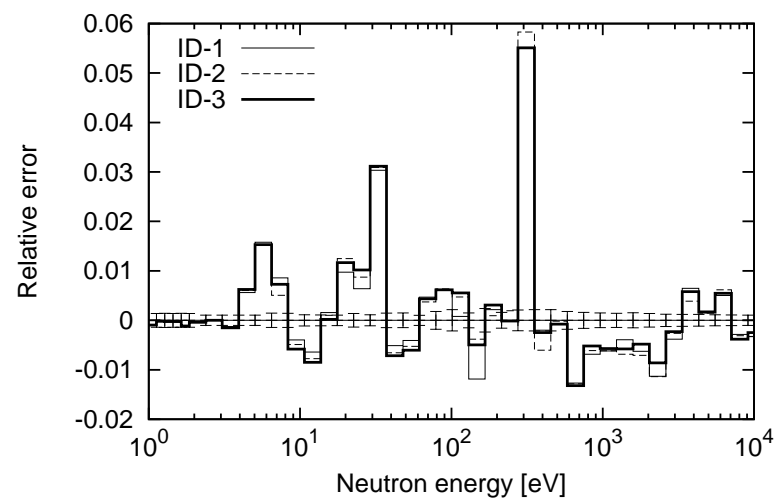

(c) BWR-UO2

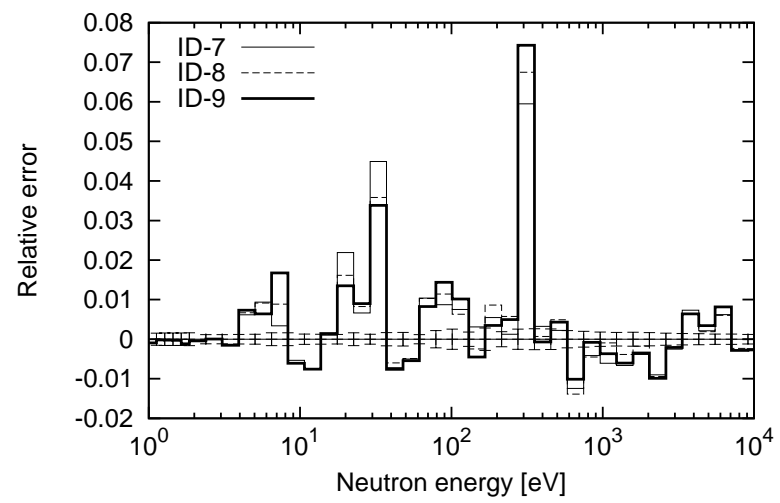

(b) PWR-MOX

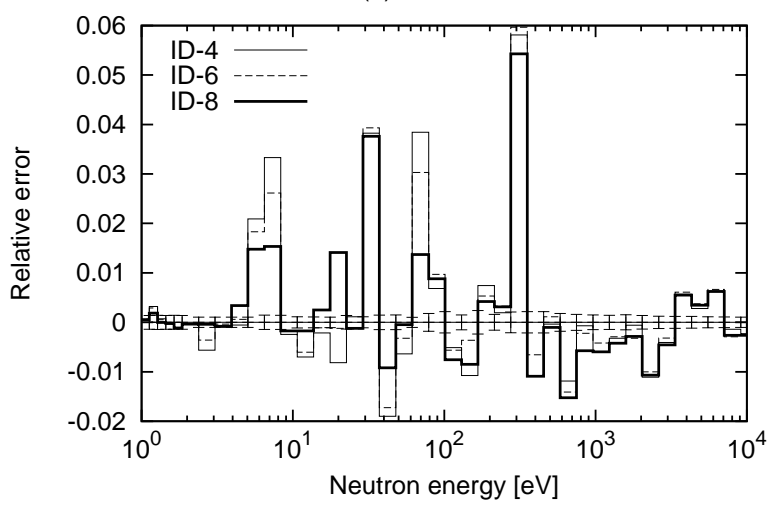

(d) BWR-MOX

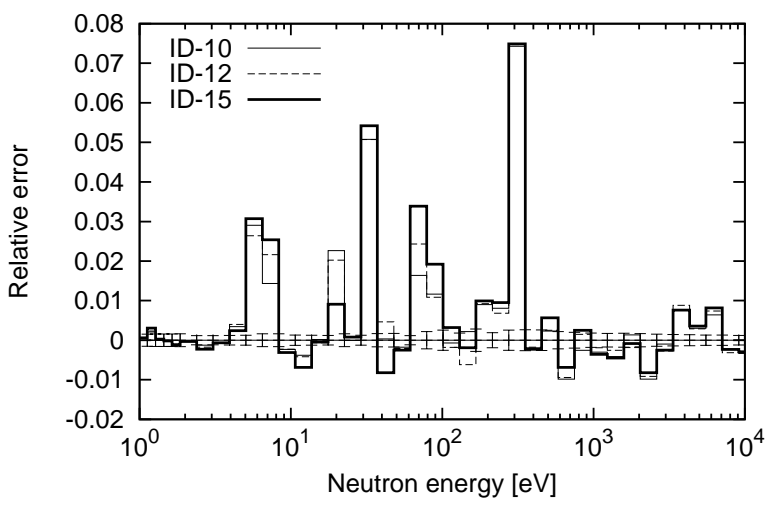

Figure 10: Reproduction errors of energy-averaged uranium-238 capture cross sections 
(a) UO2

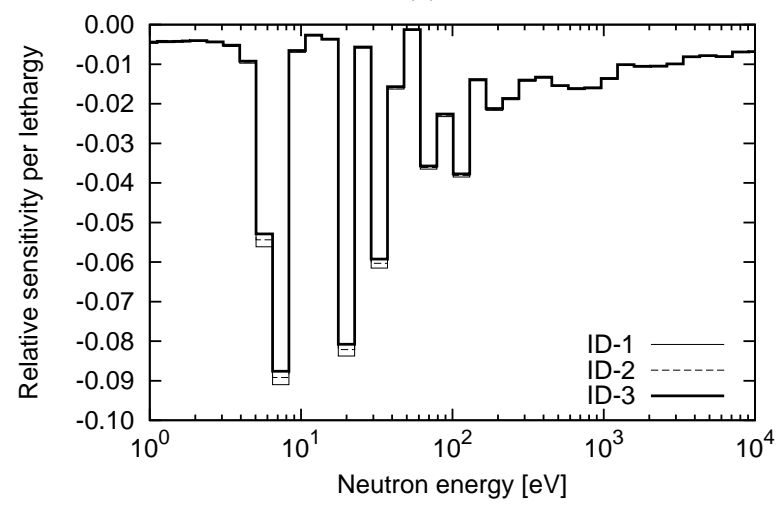

(b) MOX

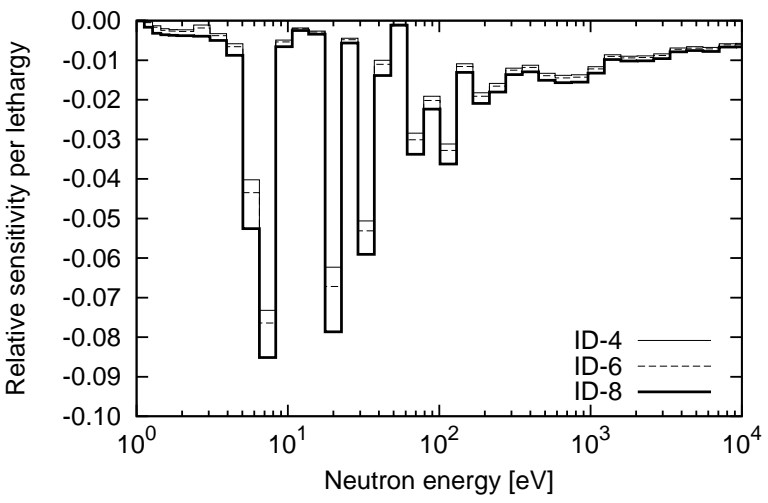

Figure 11: Relative sensitivity of $k_{\infty}$ to uranium-238 capture cross sections of PWR cells 
(a) PWR

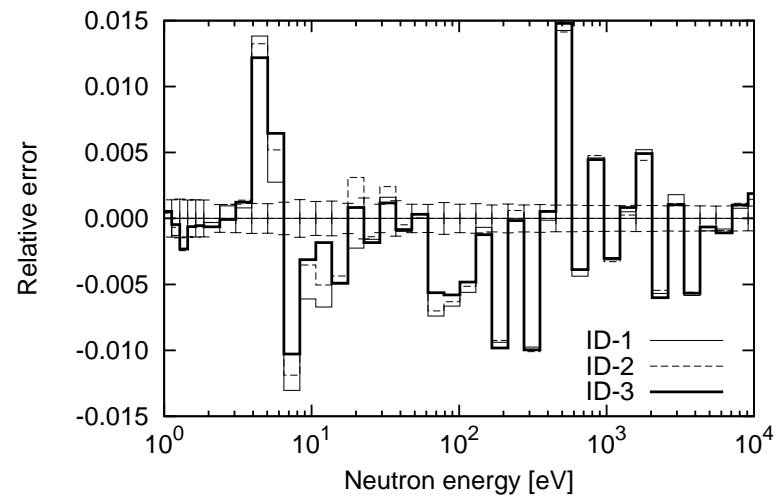

(b) BWR

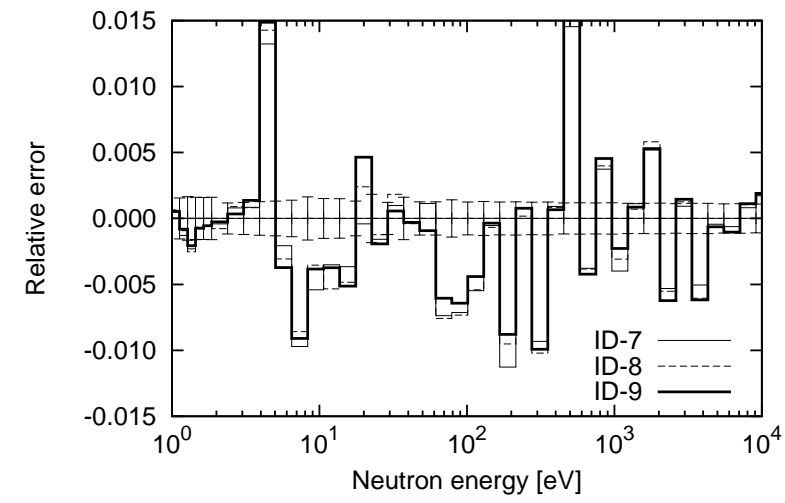

Figure 12: Reproduction errors of energy-averaged uranium-235 fission cross sections in $\mathrm{UO}_{2}$ fuel cells 
(a) PWR

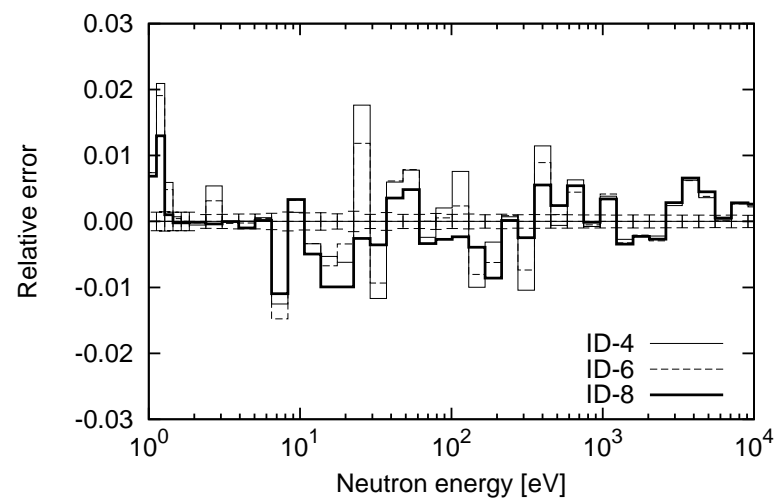

(b) BWR

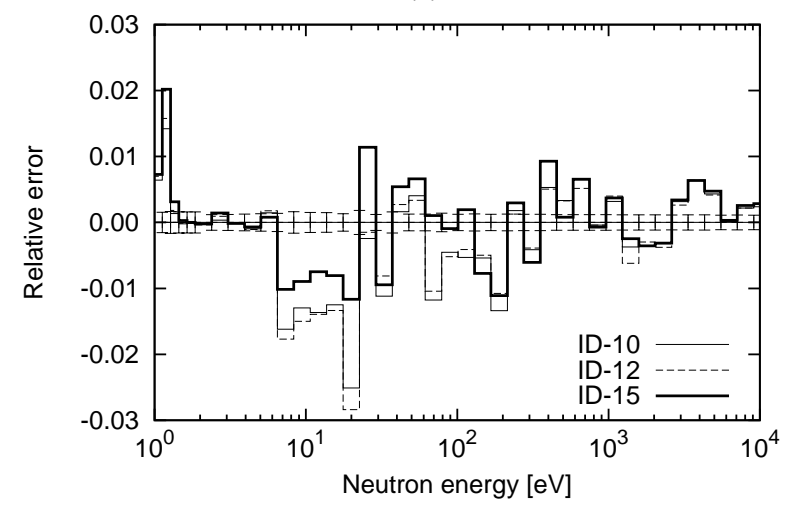

Figure 13: Reproduction errors of energy-averaged plutonium-239 fission cross sections in MOX fuel cells 


\section{List of Figure Captions}

Fig.1 Optimum Bell factors for uranium-238

Fig.2 Optimum Bell factors for uranium-235

Fig.3 Background cross sections without heterogeneous correction

Fig.4 Optimum Bell factors implemented in CBZLIB

Fig.5 Spatial discretization of fuel cell in MEC 707-group calculation

Fig.6 Reproduction errors of $k_{\infty}$ with different collapsing strategy for total cross section

Fig.7 Ratios of current-weighted (CW) uranium-238 total cross sections to flux-weighted (FW) total cross sections

Fig.8 Reproduction errors in $k_{\infty}$ against Monte Carlo reference solutions

Fig.9 Reproduction errors in $k_{\infty}$ against Monte Carlo reference solutions with different conditions

Fig.10 Reproduction errors of energy-averaged uranium-238 capture cross sections

Fig.11 Relative sensitivity of $k_{\infty}$ to uranium-238 capture cross sections of PWR cells

Fig.12 Reproduction errors of energy-averaged uranium-235 fission cross sections in $\mathrm{UO}_{2}$ fuel cells

Fig.13 Reproduction errors of energy-averaged plutonium-239 fission cross sections in MOX fuel cells 